\title{
ELLIPSOMETRIC CHANGES PRODUCED BY OXIDATION-REDUCTION POTENTIAL CYCLES APPLIED TO CADMIUM IN SODIUM HYDROXIDE SOLUTIONS
}

\author{
J. O. Zerbino, S. B. Saidman, J. R. Vilche and A. J. Arvia
}

Instituto de Investigaciones Fisicoquímicas Teóricas y Aplicadas (INIFTA), Facultad de Ciencias

Exactas, Universidad Nacional de La Plata, Sucursal 4, C. C. 16, (1900) La Plata, Argentina

(Received 9 August 1988; in revised form 13 June 1989)

\begin{abstract}
Ellipsometry data obtained for the $\mathrm{Cd}-\mathrm{NaOH}$ solution interface under the influence of potential cycles in the potential window where the $\mathrm{Cd}(\mathrm{OH})_{2}-\mathrm{CdO}$ layer can be electroformed and electroreduced, are particularly sensitive to both the layer thickness and the upper electroformation potential limit. The best fitting of the experimental results is obtained for two limiting situations. For thin anodic layers $(d \leqslant 6 \mathrm{~nm})$ the ellipsometric data fit the equation $\tilde{n}=1.55-i 0.005$, whereas for relatively thicker anodic layers $(d>7 \mathrm{~nm})$ data can be adjusted through the equation $\tilde{n}=2.6-i 0.10$. These equations are consistent with the electroformation of a hydrous cadmium oxide layer at low potentials, and a cadmium oxide layer with a lower water content which grows beneath the former one at high potentials. Otherwise, the electroreduction of the anodic oxide layer yields a cadmium overlayer which exhibits a shift of the corresponding ellipsometric data as compared to the starting cadmium surface. This shift is presumably related to the development of different metal surface textures resulting from the different electroreduction conditions.
\end{abstract}

\section{INTRODUCTION}

Passivation of $\mathrm{Cd}$ implies the formation of an anodic layer made of $\mathrm{Cd}(\mathrm{OH})_{2}$ and $\mathrm{CdO}$ which appears in general within the range of potential expected from thermodynamics[1-7]. In addition, within a certain high $\mathrm{pH}$ range the anodic reaction itself becomes complicated due to the formation of soluble $\mathrm{Cd}(\mathrm{II})$ species going into solution. Voltammetric data have shown that the passivation of $\mathrm{Cd}$ in alkaline solution implies at least two successive stages which are characterised by peaks located in well defined potential ranges $[7,8]$. Earlier work interpreted these two stages as the formation of an $\mathrm{OH}$-adsorption layer followed by a multilayer growth. However, it has been demonstrated that the properties of the passivating layer depend considerably on the way how it has been formed $[8,9]$. Likewise, the electroreduction of these layers furnishes a reduced $\mathrm{Cd}$ overlayer whose properties depend also on the way the electroreduction process has been made[10].

The present paper is devoted to investigation of the ellipsometric changes produced by oxidation- reduction potential cycles applied to a cadmium specimen immersed in sodium hydroxide solution. It should be noted that the ex situ ellipsometry data available on Cd single crystals[11-13] and on sintered and polished anhydrous $\mathrm{CdO}$ samples prepared in vacuo with different electron concentration $\left(7.4 \times 10^{18}-1.3\right.$ $\left.\times 10^{20} \mathrm{~cm}^{-3}\right)$ [14] (Table 1) are rather scattered. The present work reports ellipsometric data obtained for the cadmium-passive oxide layer system from combined electrochemical and optical measurements, and it offers the possibility to appreciate to what extent the ellipsometry data available in the literature can be applicable for the hydrous cadmium hydroxide-hydrous cadmium oxide mixtures in the passivating layer. On the basis of these results the most likely structure of the passivating layer can be envisaged.

\section{EXPERIMENTAL}

The working electrode was made from a piece of high purity poIycrystalline cadmium rod ("Specpure", Johnson Matthey Chemicals Ltd.) which was axially mounted into a PTFE cylindrical holder to offer a circular area of $0.50 \mathrm{~cm}^{2}$ apparent area. The working electrode was horizontally placed in the cell. The latter was properly designed for ellipsometric measurements. The counter electrode was a spiral shaped platinum wire. A hydrogen electrode at the electrolyte concentration was used as reference electrode, although potentials in the text are referred to the nhe scale.

The electrolyte solutions consisted of $x \mathrm{M} \mathrm{NaOH}$ $(0.01 \leqslant x \leqslant 1)$ and they were prepared from analytical grade (p.a. Merck) reagent and thrice distilled water. The solutions were continuously kept under purified

Table 1. Ellipsometric data for $\mathrm{Cd}$ and $\mathrm{CdO}$ taken from the literature

\begin{tabular}{ccccc}
\hline Substrate & $n$ & $k$ & $\lambda / \mathrm{nm}$ & Reference \\
\hline \multirow{2}{*}{ Cd } & 1.18 & 3.61 & 551.0 & {$[11]$} \\
& 0.93 & 4.98 & 546.0 & {$[12]$} \\
& 0.49 & 4.15 & 620.0 & {$[11]$} \\
& 1.97 & 4.71 & 62.8 & {$[13]$} \\
& 1.96 & 6.86 & 632.8 & \\
CdO & 2.68 & 0.41 & 546.0 & {$[14]$} \\
& 2.47 & 0.06 & & \\
\hline
\end{tabular}


$\mathrm{N}_{2}$ gas saturation. The cell was mounted in a Rudolph Research type 437-02/200 B manual ellipsometer (maximum resolution $0.01^{\circ}$ ) equipped with a $150 \mathrm{~W}$ tungsten lamp with a filter $(546.1 \mathrm{~nm})$ and a RCA IP121 photomultiplier. The incidence light beam angle was fixed at $69^{\circ}$ and that of the compensator at $135^{\circ}$. Runs were made at $25^{\circ} \mathrm{C}$. Before each measurement the working electrode was gradually polished starting with 600 grade emery paper and finished with $0.05 \mu$ grit alumina-water suspension on polishing cloth (Microcloth). Finally, it was repeatedly rinsed with thrice distilled water and held for $1 \mathrm{~min}$ in the HER potential range. From the readings, $P_{0}$ at the polariser, and $A_{\mathrm{o}}$ at the analyser, the values of $n_{\mathrm{s}}$, the refractive index, and $k_{s}$, the absorption coefficient, of the initially polished $\mathrm{Cd}$ surface were obtained. These values agree with those previously reported in the literature[11-13].

The working electrode was subjected to different simple triangular potential scans at a certain sweep rate, $v$, either continuously between the anodic, $E_{\mathrm{s}, \mathrm{a}}$, and the cathodic, $E_{s, c}$, switching potential, or by increasing $E_{\mathbf{s , a}}$ stepwise during the successive scans. Potential steps and triangular potential scans differently combined were also employed.

The ellipsometric readings, $P$ and $A$, were made under extinction conditions at both $E_{\mathrm{s}, \mathrm{c}}$ and $E_{\mathrm{s}, \mathrm{a}}$ including either a holding time at these potentials or after the current was switched off different times at $E_{\text {s.a. }}$. Ellipsometric readings were also made under dynamic conditions by using either a value of $v$ sufficiently small to neglect any change in the amount

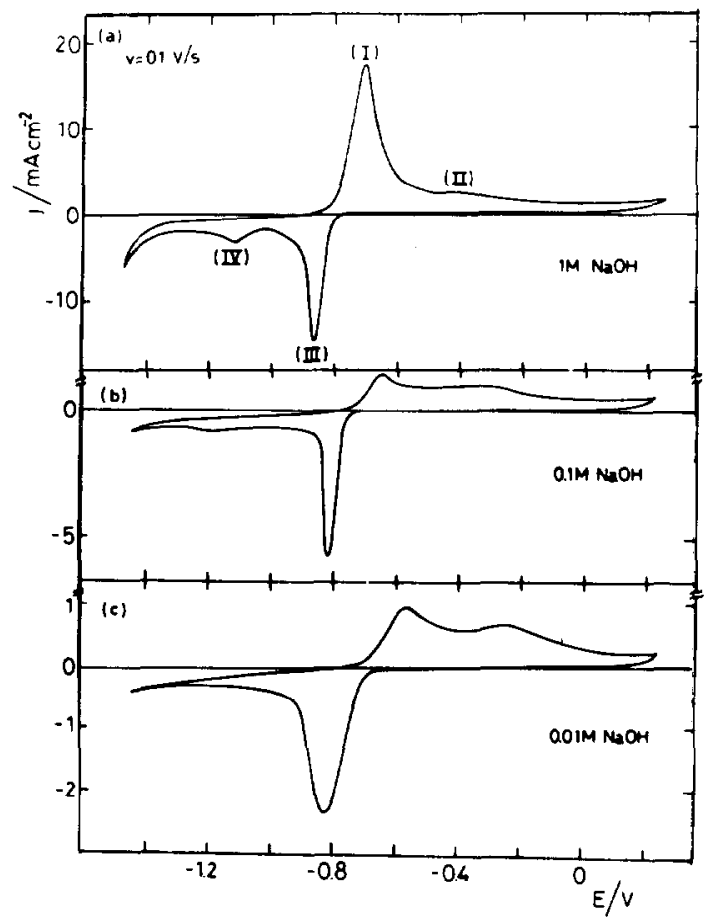

Fig. 1. Influence of the $\mathrm{NaOH}$ concentration on the stabilized voltammograms obtained at $v=0.1 \mathrm{Vs}^{-1}$ between $E_{\mathrm{s}, \mathrm{c}}=-1.46 \mathrm{~V}$ and $E_{\mathrm{s}, \mathrm{a}}=0.24 \mathrm{~V}$ : (a) 1 ; (b) 0.1 ; (c) $0.01 \mathrm{M}$ $\mathrm{NaOH}$

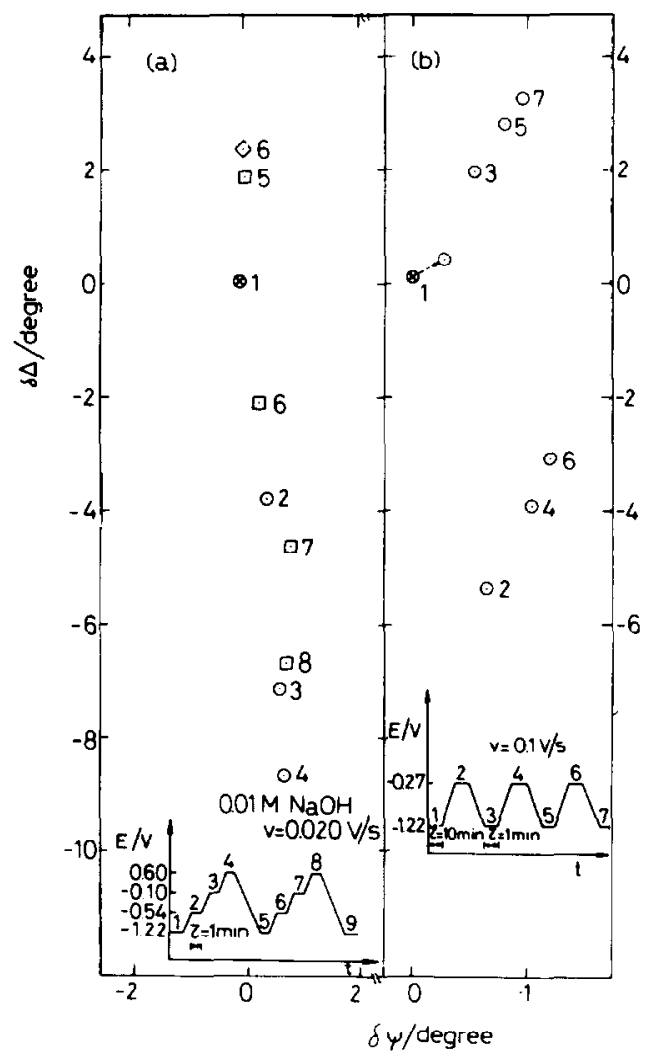

Fig. 2. Ellipsometric $(\delta \Delta v s \delta \psi)$ plots measured in $0.01 \mathrm{M}$ $\mathrm{NaOH}$ by using the potential/time programmes indicated in the figure: (a) $E_{1}=E_{5}=E_{9}=-1.22 \mathrm{~V}, E_{2}=E_{6}=-0.54 \mathrm{~V}$, $E_{3}=E_{7}=-0.10 \mathrm{~V}$, and $E_{4}=E_{8}=0.60 \mathrm{~V}$; (b) $E_{1}=E_{3}=$ $E_{5}=E_{7}=-1.22 \mathrm{~V}$, and $E_{2}=E_{4}=E_{6}=-0.27 \mathrm{~V}$. (ब) cadmium electrode held $1 \mathrm{~min}$ at $-1.22 \mathrm{~V}$.

of the oxide layer during the measurement, or at short times by following the change of $P$ throughout the variation of the reflected light intensity $[15,16]$. Ellipsometric data were depicted as $\Delta v s \psi$, and $\delta \Delta v s$ $\delta \psi$ plots, where $\delta \Delta=\Delta-\Delta_{0}$ and $\delta \psi=\psi-\psi_{0}$.

\section{RESULTS}

The voltammograms obtained for polycrystalline $\mathrm{Cd}$ electrodes in $0.01-1 \mathrm{M} \mathrm{NaOH}$ at $v=0.1 \mathrm{Vs}^{-1}$ between $E_{\mathrm{s}, \mathrm{c}}=-1.46 \mathrm{~V}$ and $E_{\mathrm{s}, \mathrm{a}}=0.24 \mathrm{~V}$, show up two anodic peaks (I and II) and two cathodic peaks (III and IV) (Fig. 1). The relative contributions of peaks I and III as compared to peaks II and IV diminish considerably as the $\mathrm{pH}$ of the solution decreases. Nevertheless, in all cases the initiation of both the layer formation and its electroreduction lies very close to the equilibrium potential of the $\mathrm{Cd}-\mathrm{Cd}(\mathrm{OH})_{2}$ redox couple at the solution $\mathrm{pH}\left(E^{\circ} / \mathrm{V}\right.$ $=0.015-0.06 \mathrm{pH}$ ). Furthermore, for $0.01 \mathrm{M} \mathrm{NaOH}$ the overall anodic to cathodic voltammetric charge ratio approaches 1 , although this ratio increases with the $\mathrm{NaOH}$ concentration.

The ellipsometric data plotted as $\delta \Delta v s \delta \psi$, for a $\mathrm{Cd}$ electrode previously cathodised during $1 \mathrm{~min}$ at $E_{\mathrm{s}, \mathrm{c}}$ in the net HER region, immediately afterwards subjected 
to a potential sweeping from $E_{\mathrm{s}, \mathrm{c}}=-1.22 \mathrm{~V}$ up to different $E_{\mathrm{s}, \mathrm{a}}$, which is set stepwise more positively, and held at each $E_{\mathrm{s}, \mathrm{a}}$ value during $\tau=1 \mathrm{~min}$, exhibit a relatively large decrease in $\Delta$ and a slight increase in $\psi$ (Fig. 2a). The changes in $\Delta$ appear to be mainly related to the accumulation of products in the potential range of peaks I and II. The $\delta \Delta$ values obtained in the passive range from $-0.1 \mathrm{~V}$ up to $0.6 \mathrm{~V}$ are about $-1.5^{\circ}$ (see points 3 and 4 in Fig. 2a). This figure is smaller than the variation of $\delta \Delta=-7.1^{\circ}$ measured up to the onset of passivity. It should also be noticed that the value of $\Delta$ read at $E_{\mathrm{s}, \mathrm{c}}=-1.22 \mathrm{~V}$ at the end of the experiment, exceeds $\mathrm{ca} 2^{\circ}$ the value initially obtained for the freshly polished $\mathrm{Cd}$ surface (compare points 1 and 5 in Fig. 2a). This shift in the value of $\Delta$ appears when any pair of points of the two cycles are considered. These results show that for the perturbation conditions employed in Fig. 2a, the successive accumulation of product is mainly responsible for the change in $\Delta$, although there is also a minor contribution related to the proper electroreduced Cd overlayer. The $\delta \Delta v s \delta \psi$ plot obtained by setting $E_{\mathrm{s}, \mathrm{a}}$ in the potential range of peak $\mathrm{II}$, ie $E_{\mathrm{s}, \mathrm{a}}=-0.27 \mathrm{~V}$ where a thicker layer is formed (Fig. 2b), shows a different trend, as in this case both $\Delta$ and $\psi$ change along the electrochemical process, although the greatest shift is that of $\Delta$. The ellipsometric data resulting for $v$ $=0.0015 \mathrm{~V} \mathrm{~s}^{1}, E_{\mathrm{s}, \mathrm{c}}=-1.75 \mathrm{~V}$ and $E_{\mathrm{s}, \mathrm{a}}=-0.10 \mathrm{~V}$ (Fig. 3a), show that independently of the potential

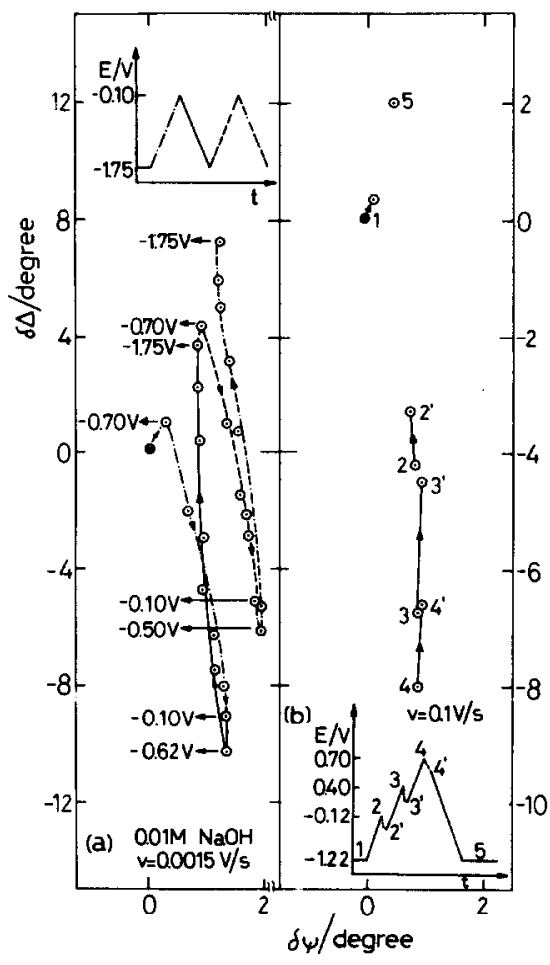

Fig. 3. (a) $\delta \Delta$ vs $\delta \psi$ plot under extinction conditions measured in $0.01 \mathrm{M} \mathrm{NaOH}$ at $\mathrm{v}=0.0015 \mathrm{Vs}^{-1}$ between $E=$ $-1.75 \mathrm{~V}$ and $E_{\mathrm{s}, \mathrm{a}}=-0.10 \mathrm{~V}$; (b) $\delta \Delta$ vs $\delta \psi$ plot made with data resulting after $0.5 \mathrm{~min}$ (points 2, 3 and 4) and $20 \mathrm{~min}$ (points $2^{\prime}, 3^{\prime}$, and $4^{\prime}$ ) current switched off from different potentials during STPS at $\mathrm{v}=0.1 \mathrm{~V} \mathrm{~s}^{-1}$ according to the potential programme indicated in the figure. sweep direction, $\Delta$ increases when a net electroreduction current is observed, and it decreases when a net electrooxidation current is recorded. Likewise, the increase of $\psi$ during the anodic reaction at low $v$ becomes greater than at high $v$, as it should be expected from the amount of anodic product accumulated at different $v$ values. Otherwise, measurements performed by including short potential scans and current interruption periods at different potentials show up an increase in $\Delta$ due to the progressive growth of the passivating layer (Fig. 3b). These results indicate that the ellipsometric response of the system follows the potential sweep but with a clear hysteresis presumably due to changes produced during the open circuit period.

Further information about the entire phenomenon can be derived through dynamic ellipsometric data obtained during the voltammetric measurements involving a sequence of linear potential sweeps and current interruptions (Fig. 4). In this case the change of $\Delta$ (Fig. 4a) is mainly associated with the accumulation of anodic product (full traces 1, 2, 3, and 4), but a considerably smaller change in $\Delta$ is observed under the open circuit period (dotted lines). In principle, these results suggest that neither the optical propertics nor the thickness of the anodic layer exhibit remarkable changes under open circuit. After $2.5 \mathrm{~min}$ current interruption the rest potential lies practically at the equilibrium potential of the $\mathrm{Cd}-\mathrm{Cd}(\mathrm{OH})_{2}$ reversible electrode. The progressive accumulation of anodic products, as seen from Fig. 4b, implies a gradual change in the height ratio of peaks I and II, and the progressive decrease of the chemical dissolution of the anodic layer.

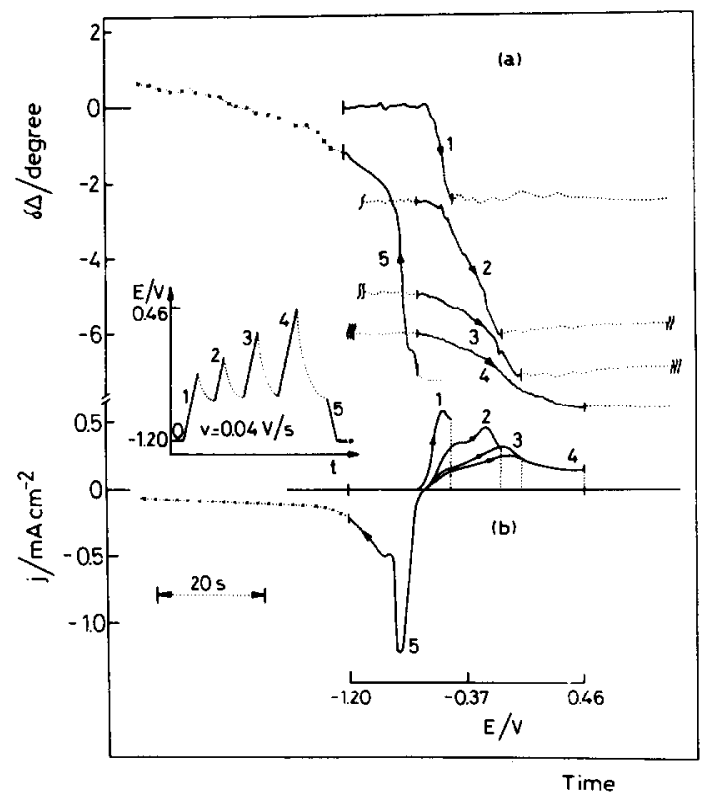

Fig. 4. Dynamic $\delta \Delta v s$ time (a) and $j$ vs $E$ (b) plots measured in $0.01 \mathrm{M} \mathrm{NaOH}$ according to the potential programme indicated in the figure. Full lines correspond to the ellipsometric and electrochemical responses at $v=0.04 \mathrm{Vs}^{-1}$. Dotted lines corresponds to open circuit conditions initiated at $E_{1}=-0.52 \mathrm{~V}, E_{2}=-0.16 \mathrm{~V}, E_{3}=0.01 \mathrm{~V}$ and $E_{4}=0.46 \mathrm{~V}$. Crossed lines correspond to the behaviour attained at $E_{\mathrm{s}, \mathrm{c}}=$ $-1.20 \mathrm{~V}$. The time of current interruption was $2.5 \mathrm{~min}$. 
On the other hand, the electroreduction of the passivating layer (Fig. $4 \mathrm{a}$, trace 5 ) implies an increase in $\Delta$, but its final value at $E_{\mathrm{s}, \mathrm{c}}=-1.20 \mathrm{~V}$ results nearly two units greater than that of the starting cadmium surface. Furthermore, the voltammetric charge density related to the electroreduction process, $Q_{c}$, after correction for the HER baseline contribution, becomes $9.0 \pm 1.0 \mathrm{mCcm}^{-2}$ (Fig. 5, curve 5), a value smaller than the anodic charge density, $Q_{\mathrm{a}}$, resulting from successive voltammetric sweeps, which is $14.5 \pm$ $1.0 \mathrm{mC} \mathrm{cm}^{-2}$ (Fig. 5, curves 1 to 4). For the latter the slope of the $Q_{\mathrm{a}}$ vs $\Delta$ plot increases according to $Q_{\mathrm{a}}$. Otherwise, by comparing curves 1 and 4 in Figs 4 and 5 , one can notice that for a constant increment of $Q_{\mathrm{a}}$, $\delta Q_{\mathrm{a}}$, the change of $\Delta$ in the potential range of peak I exceeds that resulting in the potential range of peak II.

By taking into account the changes of $\Delta$ during the open circuit periods, and by attributing those changes to a partial dissolution of the anodic product, the $Q_{\mathrm{a}}-Q_{\mathrm{c}}$ difference can be estimated from the $\delta Q v s \delta \Delta$ plots (Fig. 5). The resulting value of $Q_{\mathrm{a}}$ approaches 10 $\pm 1 \mathrm{mC} \mathrm{cm}^{-2}$, a figure which is comparable to that of $Q_{\text {c. }}$ Likewise, the variations of $\Delta$ during the electroreduction process in the potential range of peak III is considerably greater than that observed in the potential range of peak IV (Figs 4 and 5, curves 5).

The $\Delta$ vs $\psi$ plots obtained during current interruption at different potentials (Fig. 6) exhibit the greatest changes of $\Delta$ for open circuit conditions started in the $-0.5 \mathrm{~V}$ to $-0.1 \mathrm{~V}$ range (points $1-1^{\prime}$ to $3-3^{\prime}$ in Fig. 6). Otherwise, as the current is switched off at potentials set in the passive range, then the change in $\Delta$ diminishes considerably (points $4-4^{\prime}$ in Fig. 6). It should be noted that when the passivating layer growth is followed by a current interruption, the changes of $\Delta$ and $\psi$ under open circuit conditions are opposite to those observed when the anodic product is progressively accumulated (Fig. 6).

During the prolonged cathodisation of a freshly polished Cd electrode in $0.1 \mathrm{M} \mathrm{NaOH}$ the current associated with the HER increases considerably, but

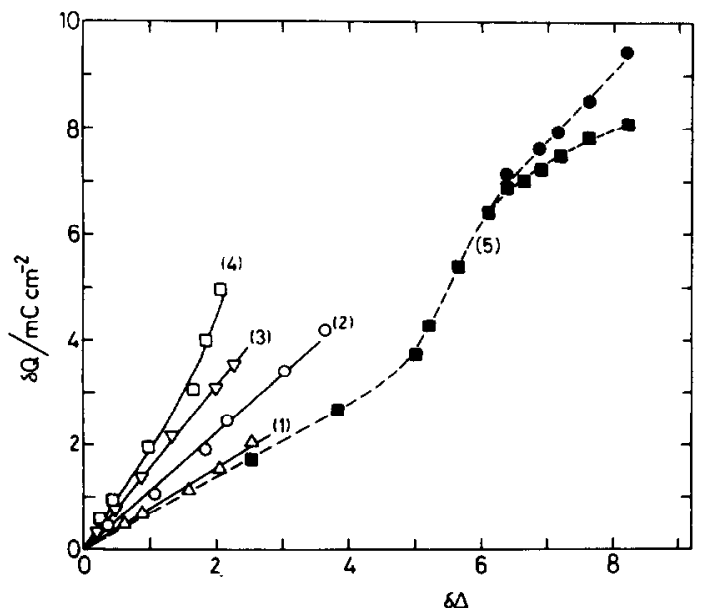

Fig. 5. $\delta \mathrm{Q} v s \delta$ plot obtained during the positive (open points, full lines 1 to 4) and negative (full points, dashed line 5) going potential scans according to the potential programme indicated in Fig. 4. Dotted curve corresponds to the cathodic charge after the correction for the HER charge.

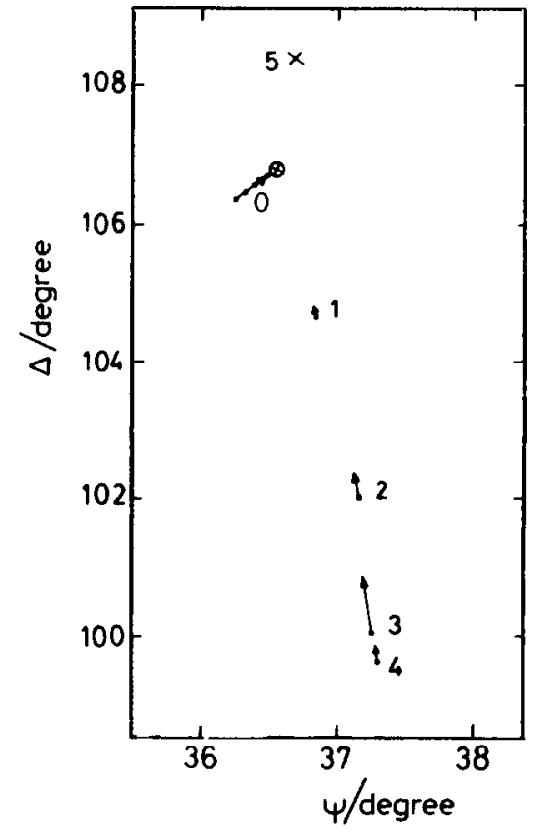

Fig. 6. $\Delta$ vs $\psi$ plot corresponding to the ellipsometric readings after $0.5 \mathrm{~min}$ (points 1 to 4 ) and $2 \mathrm{~min}$ (points $1^{\prime}$ to $4^{\prime}$ ) under open circuit conditions from measurements indicated in Fig. 4. Points 0 and 5 correspond to results at $E_{\mathrm{a}, \mathrm{c}}$ before and after the potential scan, respectively.

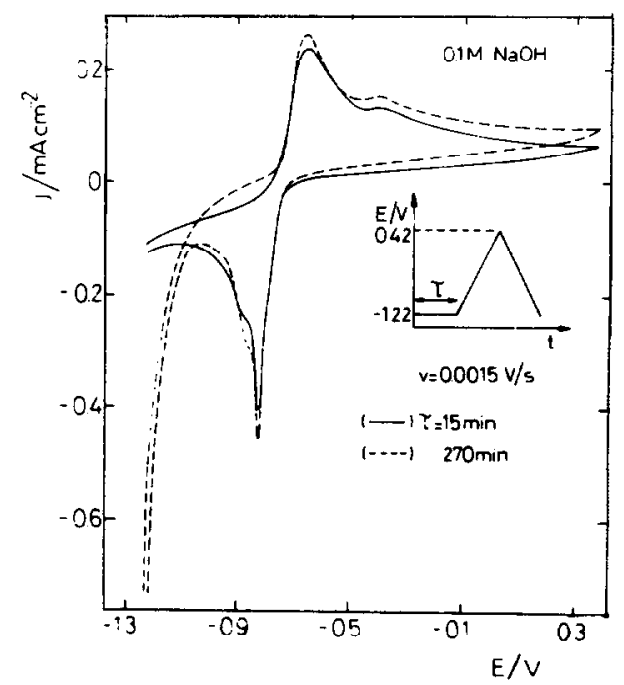

Fig. 7. Influence of the cathodisation time, $\tau$, at $E_{s, c}=$ $-1.22 \mathrm{~V}$ on the voltammograms obtained in $0.1 \mathrm{M} \mathrm{NaOH}$ at $v=0.0015 \mathrm{~V} \mathrm{~s}^{-1}$. $(\longrightarrow) \tau=15 \mathrm{~min} ;(--) \tau=270 \mathrm{~min}$.

in contrast, the voltammogram obtained in the potential range of peaks I, II, III, and IV shows up only slight changes (Fig. 7). For an initial cathodisation at $E_{\mathrm{s}, \mathrm{c}}=-1.22 \mathrm{~V}$, both $\Delta$ and $\psi$ decrease as the cathodic current increases (Fig. 8). Under these circumstances, the $\Delta v s \psi$ plots at $E_{\mathrm{s}, \mathrm{c}}$ move in the reverse direction to that shown in Figs 2,3 and 6. However, the change of the ellipsometric parameters observed during the potential sweep at $v=0.0015 \mathrm{~V} \mathrm{~s}^{-1}$ (Fig. 8) resembles those depicted in Figs 2, 3, and 6. In principle, this 


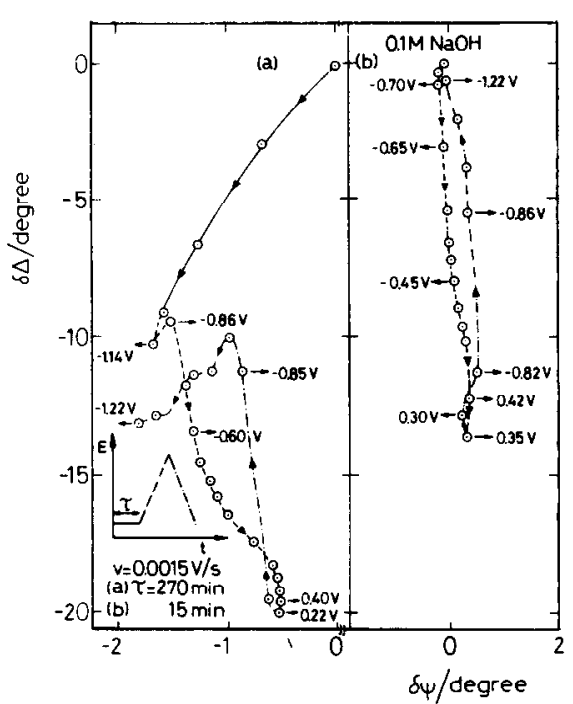

Fig. 8. $\delta \Delta v s \delta \psi$ plots measured in $0.1 \mathrm{M} \mathrm{NaOH}$ during both the cathodisation time $\tau$ (full lines) and the potential scan at $v$ $=0.0015 \mathrm{~V} \mathrm{~s}^{-1}$ (dashed lines) after $\tau=270 \mathrm{~min}$ (a) and $\tau$ $=15 \mathrm{~min}(\mathrm{~b})$.

means that both the voltammetric (Fig. 7) and ellipsometric (Fig. 8) data for $E>-1.0 \mathrm{~V}$ are not influenced by the cathodisation time, $\tau$, at $E_{\mathrm{s}, \mathrm{c}}$, although some hysteresis in the $\Delta v s \psi$ plot can be noticed for large values of $\tau$.

Similar measurements performed in $1 \mathrm{M} \mathrm{NaOH}$ show up a decrease of $\psi$ as the passivity potential range is reached, ie for $E>-0.2 \mathrm{~V}$ (Fig. 9), whereas practically no hysteresis appears during the electroreduction process for $E<-0.8 \mathrm{~V}$.

\section{DISCUSSION}

The influence of a structural parameter like $d$, the passive layer thickness, on the experimental $\Delta v s Q$ and the $\Delta v s \psi$ plots (Figs 5 and 6 ) can be envisaged through the $\Delta v s d$, and the $\psi v s d$ plots resulting from models in order to obtain a $Q$ (either $Q_{\mathrm{a}}$ or $Q_{\mathrm{c}}$ ) $v s d$ plot directly comparable to experimental data. For such a purpose let us consider the simplest situation, that is, a passive layer which grows uniformly at a constant thickness over the entire electrode surface.

The formation of $\mathrm{CdO}$ has been demonstrated by different optical methods[1-4], and its contribution to the anodic layer thickness becomes more important as $v$ decreases and the solution $\mathrm{pH}$ increases. However, in a previous work[8] it was shown that the nonstationary behaviour of cadmium in alkaline solutions comprised the formation of soluble $\mathrm{Cd}$ (II) species from the very beginning of the electrooxidation process, as detected through the rrde technique. Likewise, soluble $\mathrm{Cd}(\mathrm{II})$ could also be found at potentials more negative than the potential of peak III, but the corresponding amount was considerably smaller than that appearing in the potential range of peaks I and II. Therefore, the first fact to be taken into account in establishing any value of $d$ from the voltammetric charge, is to evaluate the charge contribution related to soluble $\mathrm{Cd}(\mathrm{II})$. This was done by assuming that the changes of $\Delta$ during open circuit conditions (Fig. 4) are directly associated with the characteristics of the $\delta \psi$ vs $\delta \Delta$ relationships (Fig. 5, curves 1 to 4 ). In this way, points 1 to 4 in Fig. 12, after correction became points $1^{\prime}$ to $4{ }^{\prime}$. Likewise, it has been supposed that up to the potential of peak III, the electroreduction charge is free from HER contribution.

An attempt can be made to interpret the ellipsometric parameters through the single layer model for the passive layer[17], and to inspect the influence of different values of $n$ and $k$ on the $\Delta v s \psi$ plots for various values of $d$. In this case $n$ and $k$ stand for the real part of the refractive index and the absorption coefficient of the passive layer, respectively. According to the experimental data the changes in the ellipsometric parameters appear to be largely associated with changes in the average thickness of the passive layer. This implies that the value of $d$ increases when $E_{\mathrm{s}, \mathrm{a}}$ is increased and $v$ is decreased. For the purpose of situating the problem, different $\Delta$ is $\psi$ plots were calculated by using the program developed by McCrackin[17] and by taking two hypothetical extreme valucs of $n$, that is, $n=2.0$ and $n=0.4$ (Fig. 10). The theoretical diagrams depict two opposite situations for the change in $\Delta$ with $k$. Thus, for $n=2.0, \Delta$ decreases with $d$ for small values of $k$, whereas for $n$ $=0.4, \Delta$ decreases with $d$ only for relatively large values of $k$. When these hypothetical results are com-

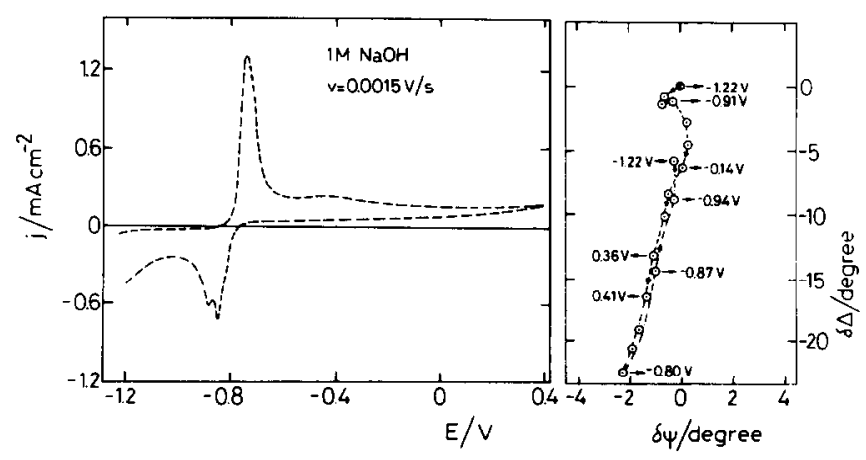

Fig. 9. Voltammogram (a) and ellipsometric plot (b) obtained in $1 \mathrm{M} \mathrm{NaOH}$ at $v=0.0015 \mathrm{~V} \mathrm{~s}^{-1}$ between $E_{\mathrm{s}, \mathrm{c}}=-1.22 \mathrm{~V}$ and $E_{\mathrm{s}, \mathrm{a}}=0.41 \mathrm{~V}$ after a cathodisation time $\tau=15 \mathrm{~min}$ at $E_{\mathrm{s}, \mathrm{c} \cdot}$ 


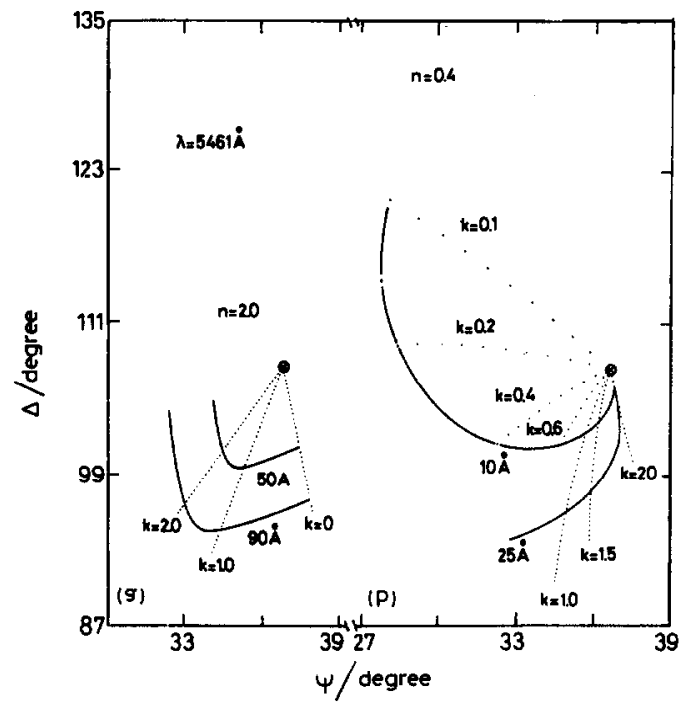

Fig. 10. Theoretical $\Delta v s \psi$ plots obtained for $n=2.0$ (a) and $n=0.4$ (b) for different values of $k$ and $d$ on the basis of a single layer model[17].

pared to the experimental data one can conclude that the best approach should be encountered for relatively large values of $n$, and as expected, for relatively small values of $k$.

Results earlier reported [8] consisting of two anodic voltammetric peaks were interpreted in terms of a time and potential dependent passive layer structure where the CdO: $\mathrm{H}_{2} \mathrm{O}$ molar ratio at the beginning of the anodic process was close to that required for a gel-like cadmium hydroxide, but at high positive potentials the passive layer becomes of increasing compactness. At this stage whether the possibility that the gel-like cadmium hydroxide layer turns into a compact layer or the latter grows directly at the metal surface as an inner layer, remains open to discussion.

According to the ellipsometric data the behaviour of the system appears to be better described in terms of two limiting situations, ie the one corresponding to thin (inner) passive layer with an average thickness $d_{1}$ $\left(d_{1} \leqslant 6 \mathrm{~nm}\right)$ formed in the potential range of peak $\mathrm{I}$, and the other one related to thicker passive layers with average diameter $\left(d=d_{1}+d_{2}>7 \mathrm{~nm}\right)$, where $d_{2}$ corresponds to the average thickness of the outer part of the passive layer. Both situations are interpreted through the single layer model by further assuming that ageing effects become negligible in the time domain of the experiments (Fig. 11). Thus, one can assume $n=1.55$ and $n=2.6$ as extreme values of $n$ in going from a thin to a thick passive layer as the electroformation potential limit increases from that of peak I up to the passivity potential range. The resulting $\Delta v s d$ plots can be discussed by comparing them to the $Q_{\mathrm{c}} v s \Delta$ plots (Fig. 12). In this case, the values of $Q_{\mathrm{c}}$ have been corrected for the amount of dissolved Cd(II) species. The $\Delta v$ s $d$ plots for $d \leqslant 6 \mathrm{~nm}$ and $d>7 \mathrm{~nm}$ depicted in Fig. 12 appear to be consistent with the existence of two distinguishable types of passive layers.

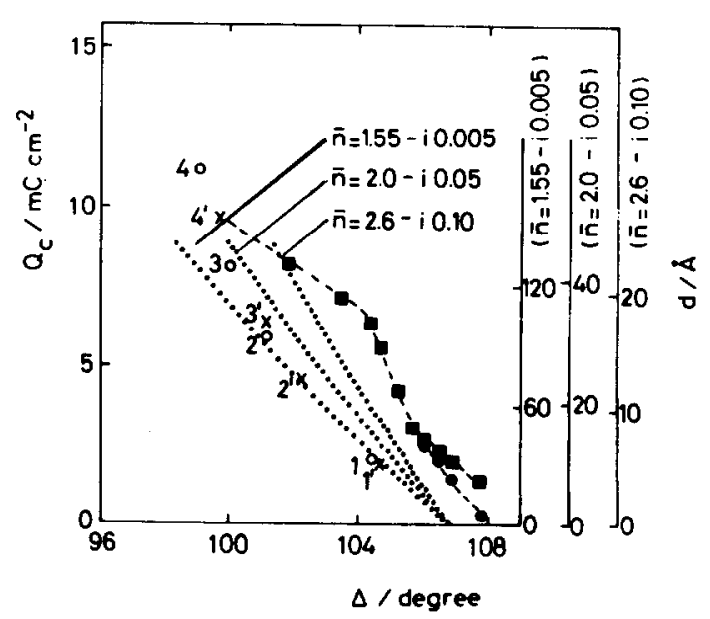

Fig. 12. Theoretical $\Delta$ vs $d$ plots calculated for different values of $n$ on the basis of a single layer model[17]. Points $1-4,1^{\prime}-4^{\prime}$, and 5 in the $Q$ vs. $\Delta$ plot correspond to the ellipsometric/electrochemical measurement indicated in Figs 4-6.

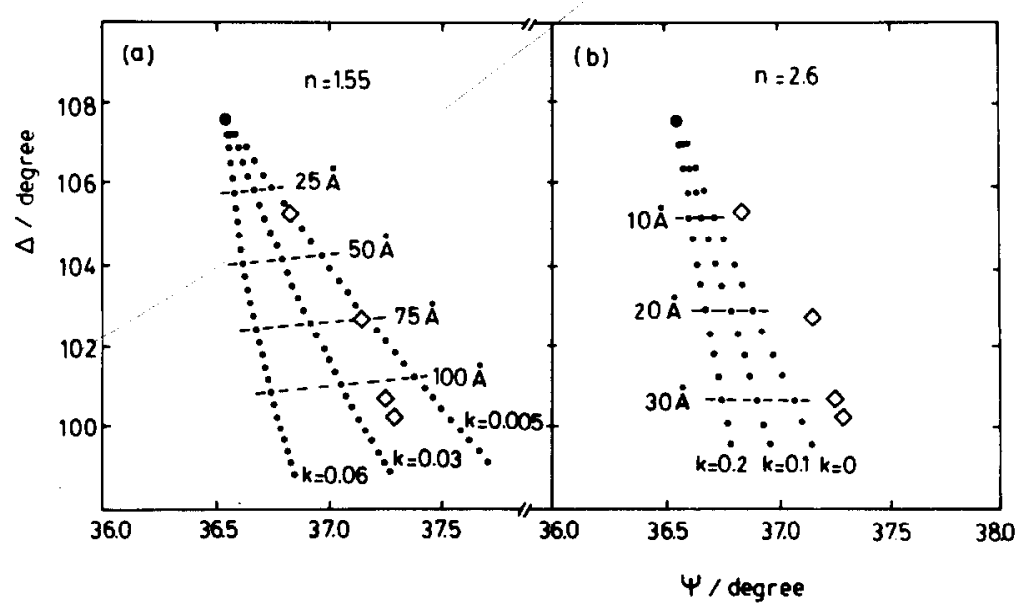

Fig. 11. Theoretical $\Delta v s \psi$ plots calculated for different $n$ and $d$ on the basis of a single layer model[17]. Experimental results ( $\diamond$ points) taken from Figs 4-6 are included. 
In the former case results fit the equation $\tilde{n}=1.55$ $-i 0.005$, and for the latter $\tilde{n}=2.6-i 0.10$. When these results are compared to those assembled in Table 1 , it can be concluded that the optical properties of the thick passive layer approach those expected for anhydrous $\mathrm{CdO}$. Therefore, there is, in principle, a reasonable coincidence between the ellipsometric data of anhydrous $\mathrm{CdO}$ prepared in vacuo [14] and the data derived from the electrochemical system, despite the different conditions prevailing in the respective measurements. It is interesting to remark that $\mathrm{CdO}$ has a very low $k$ value $546.1 \mathrm{~nm}[14]$.

From the evolution of the ellipsometric data different linear $\Delta v s d$ and $\Delta v s Q_{c}$ relationships emerge for each distinguishible type of passive layer (Fig. 12). Perhaps the interesting question brought about from Fig. 12 concerns the slopes of the $Q_{\mathrm{c}} v s \Delta$ and the $d v s \Delta$ plots for the different values of $n$. The smallest magnitude of the $\left(\delta Q_{c} / \delta \Delta\right)$ slope appears at the initial stages of the passive layer formation, and it is about the same as the $\left(\delta Q_{\mathrm{c}} / \delta \Delta\right)$ slope at the initial stages of the passive layer electroreduction. Correspondingly, the $\left(\delta Q_{\mathrm{c}} / \delta \Delta\right)$ slope increases to about twice the initial value as the thickness of the passive layer grows. The same increase can be noticed for $\left(\delta Q_{\mathrm{c}} / \delta \Delta\right)$ at the final stages of the passive layer electroreduction. These results are consistent with a structure of the passive layer made of an inner part with average thickness $d_{1}$, and outer part with average thickness $d_{2}$. This would imply that the growth of the passive layer occurs as the sum of two consecutive processes.

On the other hand, let us suppose a different situation, namely, that the passive layer involves a composite structure made of $\mathrm{CdO}, \mathrm{Cd}(\mathrm{OH})_{2}$, and electrolyte. In this case it is possible, in principle, to evaluate the effective optical constants ( $n$ and $k$ ) through either the effective medium or the Maxwell-Garnett theories[18]. Both theories predict linear dependences of $n$ and $k$ on the volume fraction of each component. For the case of one component such as $\mathrm{CdO}$, different layer thicknesses with different volumc fractions of $\mathrm{CdO}$, can store the same amount of charge. In this case, the effective optical indices follow the equations:

$$
\left(n_{1}-n_{0}\right) d_{1}=\left(n_{2}-n_{0}\right) d_{2}
$$

and:

$$
k_{1} d_{1}=k_{2} d_{2}
$$

where $n_{1}$ and $n_{2}$, and $k_{1}$ and $k_{2}$ are the effective optical indices and effective absorption coefficients of the inner and the outer layer parts, and $n_{0}$ is the optical index of the electrolyte solution. Then, the $\Delta v s d$ plot (dotted lines in Fig. 12) should be read as $\Delta v s\left(n-n_{0}\right) d$ plots for three values of $n$ where $n=n_{1}, d \Rightarrow d_{1}$ for thin layers, and $\tilde{n} \Rightarrow n_{2}$ and $d \Rightarrow d_{2}+d_{1}$ as the layer thickness increases. From these plots one can observe that $\delta Q_{\mathrm{c}} / \delta \Delta$ and the $\delta d / \delta \Delta=\delta\left(n-n_{0}\right) d / \delta \Delta$ slopes tend to coincide for low values of $Q_{\mathrm{c}}$ and $\tilde{n}=1.55-i 0.005$ (points $1,1^{\prime}, 2$, and $2^{\prime}$ in Fig. 12). Likewise, for large values of $Q_{\mathrm{c}}$ and $\tilde{n}=2.6-i 0.10$, the coincidence is again attained but for points $2,3,3^{\prime}, 4$, and $4^{\prime}$ in Fig. 12. Therefore, the model indicates that the slope of the $Q_{\mathrm{c}}$ $v s . \Delta$ and $d v s \Delta$ plots should behave in such a way that the growth of the outer layer behaves as an independent event to the previous growth of the inner layer.
This behaviour is illustrated in Fig. 13 through the slopes of the $\psi$ vs $\Delta$ plots.

If one admits, in agreement with previous kinetic data [8], that the outer layer mainly involves $\mathrm{Cd}(\mathrm{OH})_{2}$ species, and that $\mathrm{CdO}$ species predominate at the inner layer, one can relate the changes in the $(\delta \mathrm{Q} / \delta \Delta)$ slope to an electrochemical reaction in which either $\mathrm{Cd}(\mathrm{OH})_{2}$ or $\mathrm{CdO}$ species enter as reactant or product for the cathodic or anodic reaction, respectively. As the densities of $\mathrm{Cd}(\mathrm{OH})_{2}$ and $\mathrm{CdO}$ are $4.8 \mathrm{~g} \mathrm{~cm}^{-3}$ and $6.95 \mathrm{~g} \mathrm{~cm}^{-3}$, respectively[19] the formation of $\mathrm{Cd}(\mathrm{OH})_{2}$ should imply an increase in $d$ equal to $1.93 \mathrm{~nm} \mathrm{mC}^{-1}$, whereas for $\mathrm{CdO}$ it should correspond to $1.33 \mathrm{~nm} \mathrm{mC}-1$, the ratio of these values being 1.45 . This figure is not far from the ratio of the two $\left(\delta Q_{\mathrm{c}} / \delta \Delta\right)$ slopes resulting from Fig. 12. Therefore, the ellipsometric data is consistent with the fact that initial layer consists of hydrous $\mathrm{Cd}(\mathrm{OH})_{2}$. Then, the thick passive layers resulting at more passive potentials, is predominantly constituted by the inner $\mathrm{CdO}$ layer and at these potentials both layers determines the ellipsometric behaviour of the electrochemical system (Fig. 13).

From data depicted in Fig. 11 further interesting conclusions can be derived. Firstly, when the passive layer growth is interrupted under open circuit conditions, the shift of the ellipsometric data goes in the direction expected for a slight decrease in $d$, perhaps through a decrease in the water content of the hydrous oxide layer due either to open circuit ageing effects or to a dissolution process. Another interesting feature concerns the deviations in the $Q_{\mathrm{c}}$ vs $\Delta$ plots at the end of the electroreduction process in $0.01 \mathrm{M} \mathrm{NaOH}$. This drift in $\psi$ and $\Delta$ values for the electroreduced cadmium overlayer as compared to the initial metal surface is opposite to that which might be expected for the metal surface remaining still covered by a small fraction of the passive layer, or by any adsorbate. But, on the other hand, as the drift in the ellipsometric data can be correlated to the enhancement of the HER on the electroreduced cadmium overlayer [10], it is probably that these effects could be understood in terms of the

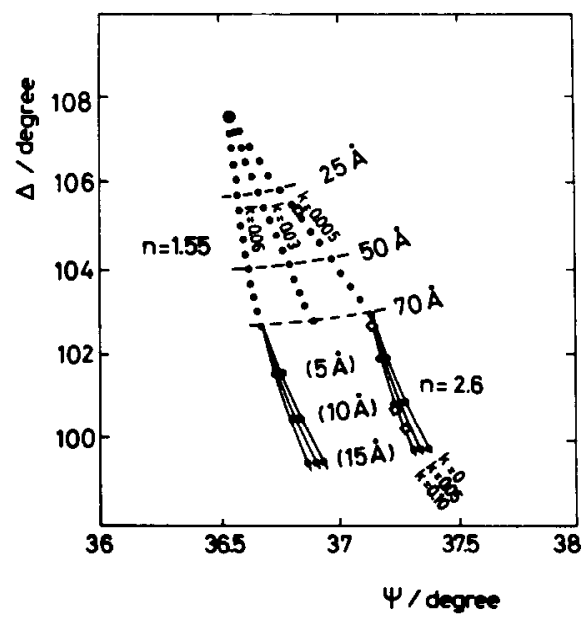

Fig. 13. Theoretical $\Delta v s \psi$ plots calculated for a film formed in two stages, the first one corresponds to an initial layer ( $n=1.55$ and different $k$ values) and the second one is associated with a layer obtained for $n=2.6$ and different $k$ values. 
development of some microporosity at the cadmium overlayer as it has already been observed for $\mathbf{A g}$ electrode[20], or through a change in the optical properties of the substrate. This question, however, can not be definitely established with the present results due to the complex structure of the electrochemical system.

\section{CONCLUSIONS}

The ellipsometric data of $\mathrm{Cd}(\mathrm{OH})_{2}-\mathrm{CdO}$ layers on cadmium in alkaline solution change considerably on the passive layer thickness. Likewise, the passive layer thickness depends on the rate and limit of the anodisation treatment.

In agreement with voltammetric data, the ellipsometric results at $\lambda=546.1 \mathrm{~nm}$ confirm that both the electroformation of the anodic layer as well as its electroreduction involve two distinguishable situations depending whether $d \leqslant 6 \mathrm{~nm}$ or $d>7 \mathrm{~nm}$. Accordingly, for $d \leqslant 6 \mathrm{~nm}$ one derives $\tilde{n}=1.55-i 0.005$ and for $d>7 \mathrm{~nm}, \tilde{n}=2.6-i 0.10$. These figures are consistent with the few data reported in the literature for $\mathrm{CdO}$ (see Table 1). There is a good correspondence between the ellipsometric data of anhydrous $\mathrm{CdO}$ prepared in vacuo and data derived from the electrochemical system. In turn, the cathodic reaction can be conceived as beginning at a layer with the same characteristics of the anodic layer initially formed, and then continuing at the rest of the layer.

Under open circuit the ellipsometric parameters of the anodic layer shift in the direction expected for a decrease in layer thickness. This fact can be associated with ageing effects presumably resulting from a slight decrease in the water content of the anodic layer. The ellipsometric parameters of the electroreduced $\mathrm{Cd}$ overlayer are different from those of the initial surface. This fact is accompanied by an enhancement of the HER.

Acknowledgement-This research project was financially supported by the Consejo Nacional de Investigaciones Cientificas y Técnicas and the Comisión de Investigaciones Científicas de la Provincia de Buenos Aires.

\section{REFERENCES}

1. P. C. Milner and V. B. Thomas, in Advances in Electrochemistry and Electrochemical Engineering (Edited by C. W. Tobias), Vol. 5, pp. 1-86, Interscience, New York (1967).

2. S. V. Falk and A. J. Salkind, Alkaline Storage Batteries, Wiley, New York (1969).

3. R. J. Lathan and N. A. Hampson, in Encyclopedia of the Electrochemistry of the Elements (Edited by A. J. Bard), Vol. 1, pp. 155-233, Marcel Dekker, New York (1973).

4. R. D. Armstrong, K. Edmondson and G. D. West, in Specialist Periodical Reports on Electrochemistry, Vol. 4, pp. 18-32, The Chemical Society, London (1974).

5. S. Gross and R. J. Glocking, The Cadmium Electrode. A Review of the States of Research, Final Report of Boeing Aerospace Company, Document D 180-19046-2 (1976).

6. F. von Sturm, in Comprehensive Treatise of Electrochemistry, Vol. 3, (Edited by J. O' M. Bockris, B. E. Conway, E. Yeager and R. E. White), Vol. 3, pp. 385-405, Plenum Press, New York (1981).

7. R. Barnard, J. appl. Electrochem. 11, 217 (1981).

8. S. B. Saidman, J. R. Vilche and A. J. Arvia, Electrochim. Acta, 32, 395 (1987).

9. S. B. Saidman, M. Lopez Teijelo, J. R. Vilche and A. J. Arvia, $4^{\text {th }}$ Sym. Bras. Electroquim. Electrical., pp. 87-98 (1984).

10. S. B. Saidman, J. R. Vilche and A. J. Arvia, J. appl. Electrochem. 18, 761 (1988).

11. A. P. Lenhan and D. M. Treherne, Proc. Phys. Soc. 83, 1059 (1964).

12. R. H. W. Graves and A. P. Lenhan, J. Opt. Soc. Am. 58, 884 (1968).

13. M. Gauch and G. Quentel, Surf. Sci. 108, 617 (1981).

14. H. Finkenrath and M. von Ortenberg, Z. Angew. Phys. 23, 323 (1967).

15. V. Brusic, M. Genshaw and B. Cahan, J. opt. Soc. 9, 1634 (1970).

16. J. O'M. Bockris, M. Genshaw and V. Brusic, Symp. Faraday Soc. 4, 177 (1970).

17. F. L. Mc Crackin, Nat. Bur. Stand. Tech. Note 479, U.S. Govt. Printing Office, Washington (1969).

18. D. E. Aspres, in Handbook of Optical Constants of Solids, (Edited by E. D. Palik), Ch. 5, pp. 104-108 Academic Press, New York (1985).

19. R. C. Weast (Editor), CRC Handbook of Chemistry and Physics 67th ed, p. B-78, CRC Press, Boca Raton, FL (1987).

20. M. Lopez Teijelo, J. O. Zerbino, I. R. Vilche and A. J. Arvia, Electrochim. Acta 29, 939 (1984). 